\title{
Cloning and functional characterisation of polyunsaturated fatty acid elongases of marine and freshwater teleost fish
}

Morris K. Agaba ${ }^{1}$, Douglas R. Tocher*, Xiaozhong Zheng, Cathryn A. Dickson, James R. Dick and Alan J. Teale Institute of Aquaculture, University of Stirling, Stirling FK9 4LA, Scotland, United Kingdom

*Corresponding author: Dr Douglas $R$ Tocher Institute of Aquaculture, University of Stirling, Stirling FK9 4LA, Scotland, telephone +44 1786 467996; fax +44 1786 472133; e-mail: d.r.tocher@ stir.ac.uk

${ }^{I}$ Current address: International Livestock Research Institute, IRLI-Kenya, P.O. Box 30709, Nairobi 00100, Kenya.

Abbreviations. AA, arachidonic acid, 20:4n-6); DHA, docosahexaenoic acid, 22:6n-3); EPA, eicosapentaenoic acid, 20:5n-3); HUFA, highly unsaturated fatty acids (chain length $\geq \mathrm{C} 20$ with $\geq 3$ double bonds); ORF, open reading frame; PUFA, polyunsaturated fatty acids. 


\begin{abstract}
:
Enzymes that lengthen the carbon chain of polyunsaturated fatty acids are key to the biosynthesis of the highly unsaturated fatty acids, arachidonic, eicosapentaenoic and docosahexaenoic acids from linoleic and $\alpha$-linolenic acids. A Mortierella alpina cDNA polyunsaturated fatty acid elongase sequence identified mammalian, amphibian, zebrafish and insect expressed sequence tags (ESTs) in GenBank. Consensus primers were designed in conserved motifs and used to isolate full length cDNA from livers of several fish species by Rapid Amplification of cDNA Ends (RACE). The amplified cDNAs encoded putative open reading frames (ORFs) of 288-294 amino acids that were highly conserved among the fish species. Heterologous expression in yeast, Saccharomyces cerevisiae, demonstrated that all of the ORFs encoded elongases with the ability to lengthen polyunsaturated fatty acid substrates with chain lengths from $\mathrm{C}_{18}$ to $\mathrm{C}_{22}$ and also monounsaturated fatty acids, but not saturated fatty acids. There were differences in the functional competence of the elongases from different fish species. Most of the fish elongases showed a pattern of activity towards different fatty acid substrates in the rank order $\mathrm{C}_{18}>\mathrm{C}_{20}$ $>C_{22}$, although the tilapia and turbot elongases had similar activity towards $18: 4 n-3$ and 20:5n-3. The fish elongases generally showed greater activity or similar activities with n-3 than with n-6 homologues, with the exception of the cod enzyme which was more active towards n- 6 fatty acids.
\end{abstract}

Keywords: Fish, highly unsaturated fatty acid, elongase, cDNA, gene, chain elongation. 


\section{Introduction}

Dietary long chain polyunsaturated fatty acids (PUFA), and highly unsaturated fatty acids (HUFA), are crucial to the nutritional health, physiology and reproductivity of higher vertebrates (Burr, 1981; Simopoulos, 2000). HUFA have a well-known, essential role as components of structural phospholipids of cell membranes. Docosahexaenoic (DHA; 22:6n-3) and arachidonic (AA; 20:4n-6) acids are particularly required for proper neural development and function (Lauritzen et al., 2001), and metabolites of AA and eicosapentaenoic acid (EPA; 20:5n-3), such as leukotrienes and prostaglandins, have a wide range of signalling functions (Lands, 1993). Diseases arising from HUFA deficiency are well documented in many species (Nugent, 2004; Graham et al., 2004). In fish there are degenerative lesions in the viscera, erosions of fins and gills, and poor adaptation to low temperature (March, 1993).

As with all vertebrates, PUFA are essential components of the diet in fish, but qualitative and quantitative requirements vary with species (Sargent et al., 1995, 2002). In salmonids, dietary $\alpha$ linolenic acid (18:3n-3) and linoleic acids (18:2n-6) alone are sufficient for optimal growth. In Nile tilapia (Oreochromis nilotica), both 18:2n-6 and AA are better growth promoters than are 18:3n-3 and EPA (Sargent et al., 1995, 2002). In marine fish, the dietary requirement is for HUFA rather than shorter chain PUFA. For example, turbot (Psetta maxima), grow optimally on EPA and DHA, but not 18:3n-3 (Sargent et al., 1995, 2002). Differences in the capacity of fish species to thrive on diets containing only the $\mathrm{C}_{18}$ PUFA, 18:2n-6 and 18:3n-3, or to require dietary HUFA appear to be evolutionarily related to the nature of the fatty acid profiles present in their respective diets. Thus, fresh water fish such as tilapia whose vegetarian diet generally lacks HUFA, convert 18:2n-6 and 18:3n-3 to HUFA more efficiently than do marine species such as turbot whose predominantly carnivorous (largely piscivorous) diet is rich in HUFA (Sargent et al., 2002; Tocher 2003).

In vertebrates, biosynthesis of HUFA can proceed by sequential desaturation and elongation of 18:2n-6 and 18:3n-3. Synthesis of AA is achieved by a $\Delta 6$ desaturation of $18: 2 n-6$ to produce $\gamma$ linolenic acid (18:3n-6) that is elongated to dihomo- $\gamma$-linolenic acid (20:3n-6) and finally desaturated at the $\Delta 5$ position to produce AA (Fig.1) (Cook, 1996). The pathway for EPA synthesis from 18:3n-3 is essentially similar, but DHA synthesis requires two further elongation steps, a second $\Delta 6$ desaturation and a chain shortening step (Fig.1) (Sprecher, 2000). Experimental evidence suggests that the dependence of marine fish on dietary HUFA is caused by deficiency in the activity of one or more of the key enzymes, $\Delta 5$ and $\Delta 6$ fatty acid desaturases, and fatty acid elongases, required for HUFA biosynthesis (Sargent et al., 2002; Tocher, 2003). In vivo studies, using $\left[1-{ }^{14} \mathrm{C}\right] 18: 3 n-3$ supplied to the fish either in force-fed pellets or injected intraperitoneally, consistently showed the superiority of freshwater species over marine species in the biosynthesis of ${ }^{14} \mathrm{C}$-EPA and ${ }^{14} \mathrm{C}$-DHA (Owen et al., 
1975; Mourente and Tocher, 1993a,b, 1994). In vitro studies indicated major impairments in the HUFA synthetic pathway of cell lines from marine fish species, turbot (TF) and gilthead sea bream (SAF-1) in comparison to those of cell lines from salmonids (Ghioni et al., 1999; Tocher and Ghioni, 1999).

There are potentially profound implications of these differences in terms of animal nutrition, aquaculture and human health. Fish are the only major dietary source for humans of n-3HUFA and, with declining fisheries, farmed fish constitute an increasing proportion of the fish in the human diet (Tidwell and Allan, 2002). However, the current high use of fish oils, derived from feed grade marine fisheries, in aquaculture feeds is not sustainable, and will constrain continuing growth of aquaculture activities (Barlow, 2000). The only sustainable alternative to fish oils are vegetable oils which are rich in $\mathrm{C}_{18}$ PUFA but devoid of the n-3HUFA abundant in fish oils (Sargent et al., 2002). Therefore, there is currently considerable interest in the HUFA biosynthetic pathway in fish to determine the effectiveness with which vegetable oils can be utilized by cultured fish species (Sargent et al., 2002).

We have previously reported the cloning and functional characteristics of desaturase and elongase enzymes in the model fresh water species zebrafish (Danio rerio) (Hastings et al., 2001; Agaba et al., 2004). Zebrafish elongase when expressed in yeast (Saccharomyces cerevisiae) lengthens all the key intermediates including 18:4n-3, EPA and 22:5n-3 required for the biosynthesis of DHA. This property is consistent with the fact that zebrafish can grow well on diets rich in vegetable oils lacking DHA, and whose hepatocytes have been shown to produce labelled DHA from $\left[1-{ }^{14} \mathrm{C}\right] 18: 3 n-3$ (Tocher et al., 2001). A PUFA elongase with similar activity to the zebrafish elongase has also been cloned from Atlantic salmon (Salmo salar) (Hastings et al., 2004). Here we extend the study of fish elongases to cover five fish species, spanning four Orders. Two species, Nile tilapia (Oreochromis niloticus, Perciformes) and African catfish (Clarius gariepinus, Siluriformes), inhabit freshwater systems and can thrive well on vegetable oil diets, and three are marine species, cod (Gadus morhua, Gadiformes), gilthead seabream (Sparus aurata, Perciformes); and turbot (Pleuronectiformes) that cannot thrive on diets lacking HUFA. Thus, we report the cloning and functional characterisation of the cDNAs of PUFA elongases from species that span the range of dietary HUFA dependency observed among fish.

\section{Materials and methods}

\subsection{Cloning of fish putative elongase cDNAs}

Fish elongase cDNAs were isolated by PCR amplication of cDNA derived from liver total RNA of African catfish, Nile tilapia, turbot, gilthead seabream and Atlantic cod prepared using TRIzol® 
reagent (GibcoBRL, NY, U.S.A.). 5' and 3' Rapid Amplification of cDNA Ends (RACE) was achieved with the SMART ${ }^{\mathrm{TM}}$ RACE cDNA amplification kit (Clontech, NJ, U.S.A). For initial cDNA isolation degenerate primers were designed based on conserved motifs in the putative elongase expressed sequence tag (EST) sequences of Mortierella alpina (AF206662), Homo sapiens ELOVL5 (AF231981), Mus musculus (AF170908), Xenopus laevis (BG813561), Danio rerio (AF532782, BF157708) and Drosophila melanogaster (AI113483). The forward primers UniElo1A (C CTG TGG TGG TAY TAY TT) and UniElolAA (GAR TTY ATG GAC ACN TTC TTC TT) targeted the conserved regions VLWWYYF and EFMDTFFF, respectively and the reverse primer UniElo1B (YTG NGT RTA YTT YTT CCA CCA) targeted the conserved region WWKK. Following isolation of cDNA fragments flanked by UniElo1AA and UniElo1B, specific primers for use in 5'RACE and 3' RACE PCR were designed. All PCR products were cloned in pBlueScript SKII vector (Stratagene, La Jolla, CA, USA) and sequenced using dye terminator chemistry in a Perkin Elmer ABI-377 DNA sequencer with associated software following the manufacturer's protocols (Perkin Elmer, Applied Biosystems). The full-length sequences were assembled and translated to the conceptual amino acid sequences. Deduced amino acid sequences were aligned using ClustalX and sequence phylogenies were predicted using the Neighbour Joining method of Saitou and Nei (1987). Confidence in the resulting phylogenetic tree branch topology was measured by bootstrapping the data through 1000 iterations.

\subsection{Functional characterisation of fish elongase ORFs by heterologous expression in yeast,}

Saccharomyces cerevisiae

Functional characterisation of the genes was by expression of the open reading frames (ORFs) of the putative fatty acid elongase sequences in S. cerevisiae. Primers were designed for amplification of the ORFs of each fish elongase from the corresponding cDNA template (Table 1). These primers carried restriction sites to enable cloning into the episomal yeast expression vector pYX222 (R \& D Systems Europe Ltd., Abingdon, Oxon, U.K.). Thus, the forward primers contain the Kozak translation initiation sequence (boldface) and an EcoRI site (underlined). The reverse primers flank or contain the termination codon and have an XhoI site. After PCR using high fidelity DNA polymerase mix (Roche Diagnostics Ltd., Lewes, East Sussex, U.K.), the DNA fragments were restricted with EcoRI and XhoI and ligated into similarly treated pYX222 plasmid, which was then used to transform TOP10F' E.coli competent cells (Invitrogen Ltd, Paisley, UK) as described previously (Hastings et al., 2001). Recombinant plasmids were prepared from at least six independent E.coli colonies and, after sequence confirmation, were used to transform bakers' yeast (S. cerevisiae strain INVSc1). Yeast transformed with non-recombinant pYX222 plasmid was used as control. Transformation of the yeast with the recombinant plasmids, and yeast culture were performed as described in detail previously (Hastings et 
al., 2001). Briefly, recombinant yeast was grown in $S$. cerevisiae minimal medium ${ }^{\text {-histidine }}\left(\mathrm{SCMM}^{\text {-his }}\right)$ supplemented with the following PUFA substrates; stearidonic acid (18:4n-3), $\gamma$-linolenic acid (18:3n6), EPA (20:5n-3), AA (20:4n-6), docosapentaenoic acid (22:5n-3) and docosatetraenoic acid (22:4n6). Yeast cells were harvested, washed and dried, and total lipid extracted with ice-cold chloroform/methanol (2:1, v/v) essentially according to Folch et al. (1957). Fatty acid methyl esters (FAME) were prepared by acid-catalysed transesterification (Christie, 1982), and FAME extracted, purified by thin layer chromatography, and analysed by gas chromatography (GC) all as described previously (Hastings et al., 2001). The proportion of substrate fatty acid converted to the longer chain fatty acid product was calculated from the gas chromatograms as $100 \times$ [product area/(product area + substrate area)]. Unequivocal confirmation of fatty acid products was performed by GC-mass spectrometry of the picolinyl derivatives as described in detail previously (Hastings et al., 2001).

\subsection{Materials}

Docosapentaenoic (22:5n-3) and docosatetraenoic (22:4n-6) acids (both $>98-99 \%$ pure) were purchased from Cayman Chemical Co., Ann Arbor, U.S.A. Stearidonic (18:4n-3), $\gamma$-linolenic (18:3n6), EPA (20:5n-3) and AA (20:4n-6) acids (all >99\% pure), BHT, 1,1'-carbonyldiimidazole, 2,2dimethoxypropane, fatty acid-free BSA, galactose, 3-(hydroxymethyl)pyridine, nitrogen base, raffinose, tergitol NP-40 and uracil dropout medium were obtained from Sigma Chemical Co. Ltd., Dorset, UK. TLC $(20 \times 20 \mathrm{~cm} \times 0.25 \mathrm{~mm})$ plates precoated with silica gel 60 (without fluorescent indicator) were purchased from Merck, Darmstadt, Germany. All solvents were HPLC grade and were from Rathburn Chemicals, Peebleshire, U.K.

\section{Results}

\subsection{Sequence analyses}

A search of the public EST database identified sequences from several species including mammalian (AF231981, AF170908), avian (XM_426505), amphibian (BG813561), fish (AF532782, BF157708) and insect (AJ871925) that were related to the M. alpina PUFA elongase cDNA (AF206662). By taking advantage of highly conserved motifs in the amino acid sequences, full-length elongase transcripts were isolated for each of the target fish species with ORFs of $885,882,885,885$ and 867 base pairs for African catfish, Nile tilapia, gilthead sea bream, turbot and cod, respectively. The predicted elongase polypeptides consist of 288 - 294 amino acid residues, and include characteristic 
features of microsomal fatty acid elongases, including a single histidine box redox centre motif (HXXHH), a canonical ER retention signal (carboxyl-terminal dilysine targeting signal), and multiple transmembrane regions (Fig.2). The KXXEXXDT. QXXFLHXYHH (which contains the histidine box), NXXXHXXNYXYY and TXXQXXQ motifs are highly conserved in all PUFA elongases cloned to date (Meyer et al., 2004). The translated amino acid sequences of the elongase cDNAs cloned from the five fish species here, along with our previously cloned zebrafish and salmon sequences, are very similar to each other and share at least $61 \%$ sequence identity with the human elongase ELOVL5 (Table 2), with most of the sequence variation (insertions and substitutions) residing in the C-terminal region of the polypeptide (Fig. 2). Phylogenetic analysis by neighbour-joining comparing all the putative elongase sequences cloned from fish, along with a range (not exhaustive) of elongases from mammals, bird, insect, fungus and nematode, clustered all the fish sequences together and showed they were most similar to the mammalian (human/rodent) ELOVL5/elovl5 (rat rELO2) and ELOVL2/elovl2 (mouse Ssc2) elongase sequences (Fig.3).

\subsection{Functional characterisation}

The fish putative elongase cDNAs were functionally characterized by determining the fatty acid profiles of transformed S. cerevisiae containing either the pYX222 vector alone or the vector containing the fish elongase cDNA inserts, grown in the presence of a variety of possible fatty acid substrates including 18:4n-3 and 18:3n-6 $\left(\mathrm{C}_{18-20}\right), 20: 5 n-3$ and 20:4n-6 $\left(\mathrm{C}_{20-22}\right)$ and 22:5n-3 and 22:4n-6 $\left(\mathrm{C}_{22-24}\right)$ (see Fig.1). The fatty acid composition of the yeast transformed with the pYX222 vector alone showed the four main fatty acids normally found in S. cerevisiae, namely 16:0, 16:1n-7, 18:0 and 18:1n-9, together with whichever exogenous substrate fatty acid was added, consistent with $S$. cerevisiae not possessing PUFA elongase activity. With all the fish putative fatty acid elongase cDNAs investigated, the most prominent additional peaks were generally observed in the profile of transformed yeast grown in the presence of the $\mathrm{C}_{18}$ substrates, 18:4n-3 and 18:3n-6 (Figs. 4-7; Table 3). GC traces showing the fatty acid compositions of the yeast transformed with the pYX222 vector containing the putative fatty acid elongase cDNA inserts grown in the presence of 18:4n-3 are shown in Fig. 4. In addition to the major endogenous fatty acids (16:0, 16:1n-7, 18:0 and 18:1n-9, peaks 1-4), and the exogenously added substrate fatty acid (18:4n-3, peak 6) the traces show a major additional peak (peak 9) (Fig.4). Based on GC retention time and confirmed by GC-MS, the additional peak associated with the presence of the fish elongase cDNAs was identified as 20:4n-3, corresponding to the elongation product of 18:4n-3 (Fig.4). Other additional peaks were also noted including 22:4n-3 (peak 10), 18:1n-7 (peak 5) and 20:1n-9 and 20:1n-7 (peaks 7 and 8, respectively) (Fig.4). The fatty 
acid compositions of transformed yeast grown in the presence of 20:5n-3 are shown in Fig. 5. In addition to the endogenous yeast fatty acids and the exogenously added substrate fatty acid (20:5n-3, peak 11) the traces show a major additional peak (peak 12) confirmed by GC retention time and GCMS as 22:5n-3, the elongation product of 20:5n-3. When transformed yeast were grown in the presence of 22:5n-3 (peak 12), an additional peak (peak 13) was observed that was confirmed by GC-MS to be 24:5n-3, as illustrated in Fig.6. Although there were differences in the substrate specificities of the fish elongases, most showed a pattern of activity towards different fatty acid substrates in the rank order $\mathrm{C}_{18}$ $>\mathrm{C}_{20}>\mathrm{C}_{22}$ (Table 3). However, the tilapia and turbot elongases showed similar activity towards 18:4n3 and 20:5n-3 (Table 3). The elongase cDNA products tended to be more active towards the n-3 substrate or show similar activity with n-3 and n-6 substrates, with the exception of the cod elongase, which was more active towards the n-6 fatty acid homologues (Fig.7; Table 3).

\section{Discussion}

Here we report the cloning and functional characterisation of several cDNAs derived from genes that are responsible for the chain elongation of PUFA in a range of freshwater and marine teleost fish species. The cDNAs encode proteins that are very similar to mammalian PUFA elongases, and have all the main structural characteristics possessed by elongases derived from other systems. These include the so-called histidine box $(\mathrm{HXXHH})$, the canonical C-terminal endoplasmic reticulum (ER) retention signal, several predicted transmembrane domains and other highly conserved motifs (Meyer et al., 2004). There is remarkable conservation of the predicted amino acid sequences of the fish species, and indeed among all the vertebrates. The phylogenetic sequence analyses grouped the fish elongases into basically four clusters largely as expected based on the four main groups of modern teleosts identified in classical phylogeny with the clusters being catfish and zebrafish (Ostariophysi; siluriformes and cypriniformes), cod (Paracanthopterygii; gadiformes), trout and salmon (Salmoniformes; salmonidae), and tilapia, sea bream and turbot (Acanthopterygii; perciformes/chichlids and pleuronectiformes) (Nelson, 1994). A similar phylogenetic grouping was observed previously when the sequences of fatty acid desaturases from a similar range of teleosts were analysed (Zheng et al., 2004a). It was noteworthy that the fish PUFA elongases clustered most closely with the mammalian ELOVL5/elov15 and ELOVL2/elovl2 elongases that have been functionally characterised and shown to be PUFA elongases. They were more distant from other mammalian elongases, such as rat rELO1 (also known as rat Elov16). The latter has been shown to be predominantly a 16:0 to 18:0 elongase (Inagaki et al., 2002) that clustered with a chicken elongase (also with elovl6 homology), and human ELOVL4 and 
mouse Ssc1 elongases that are not functionally characterised, but which are presumed to be involved in sphingolipid metabolism and the synthesis of very long chain fatty acids such as 26:0 (Lagali et al., 2003; Tvrdik et al., 2000).

Studies over the last few years have shown that there is a large family of fatty acid elongases, which can be perhaps broadly classified into two main groups. Elongases with activities predominantly towards saturated (and possibly monounsaturated) fatty acids and either closely linked to fatty acid synthesis $\left(\mathrm{C}_{12}-\mathrm{C}_{16}\right)$ or sphingolipid synthesis $\left(\mathrm{C}_{22}-\mathrm{C}_{26}\right)$, and elongases with activity predominantly towards PUFA with chain lengths in the $\mathrm{C}_{18}-\mathrm{C}_{22}$ range. Functional characterisation by heterologous expression in $S$. cerevisiae showed that all the fish cDNAs were predominantly PUFA elongases with highest activity towards $\mathrm{C}_{18}$ fatty acids, but also with high activity towards $\mathrm{C}_{20}$ fatty acids and, in most cases, activity towards $\mathrm{C}_{22}$ fatty acids. The relatively loose substrate specificity of the fish elongases was highlighted by the presence of 22:4n-3 in the fatty acid profiles of $S$. cerevisiae when transformed with the catfish, tilapia, sea bream and turbot ORFs and grown in the presence of 18:4n-3, showing further elongation of 20:4n-3 produced by elongation of the exogenously added 18:4n-3. Some 24:5n3 was also produced in yeast transformed with elongases from these species when incubated with EPA (data not shown), as previously observed with zebrafish and salmon elongases (Agaba et al., 2004; Hastings et al., 2004). Recently, an elongase was cloned from rainbow trout (Oncorhynchus mykiss) and shown, along with elongases from an amphibian (Xenopus laevis) and the sea squirt (Ciona intestinalis), to accept both $\mathrm{C}_{18}$ and $\mathrm{C}_{20}$ PUFA substrates (Meyer et al., 2004).

Previously, it was shown that PUFA elongases from human (ELOVL5) and rat (rELO1/elov15) were highly active towards $C_{18}$ PUFA, but also showed significant activity towards 20:5n-3 and 20:4n6 (Leonard et al., 2000: Inagaki et al., 2002). In contrast, the elongases of the filamentous fungus $M$. alpina (GLELO) and the nematode $C$. elegans were highly active towards $\mathrm{C}_{18}$ PUFA, but showed virtually no activity towards $\mathrm{C}_{20}$ PUFA, consistent with these organisms not containing any significant level of $\mathrm{C}_{22}$ fatty acids (Parker-Barnes et al., 2000; Beaudoin et al., 2000). Two further mammalian elongases, from human (ELOVL2) and mouse (elov12/Ssc2), have been shown to possess the ability to elongate 22:5n-3 and 22:4n-6 to 24:5n-3 and 24:4n-6, respectively (Leonard et al., 2002). The mouse elov12 clone was also able to elongate $\mathrm{C}_{18}$ and $\mathrm{C}_{20}$ PUFA, whereas the human ELOVL2 clone was only active towards $\mathrm{C}_{20}$ and $\mathrm{C}_{22}$ PUFA (Leonard et al., 2002). Thus both mammals and fish have the ability to elongate $\mathrm{C}_{20}$ and $\mathrm{C}_{22}$ PUFA in addition to $\mathrm{C}_{18}$ PUFA. Humans may require two enzymes for all these elongations whereas mouse and fish appear to require only one. However, the existence of more than one PUFA elongase in human suggests that other elongases with differing substrate specificities may yet be isolated in fish. 
That fish, like mammals, can chain elongate $\mathrm{C}_{18}, \mathrm{C}_{20}$ and $\mathrm{C}_{22}$ PUFA is significant in terms of HUFA synthesis. Direct synthesis of DHA from 22:5n-3, as has been described in the marine microheterotroph, Thraustochytrium sp (Qui et al., 2001), requires desaturation of PUFA at the $\Delta 4$ position, but this activity has not been demonstrated in any vertebrate system (Tocher et al., 1998; Tocher, 2003). Alternatively, synthesis of DHA in rat liver was shown by Sprecher and co-workers to proceeded via $\mathrm{C}_{24}$ PUFA intermediates through a pathway requiring two sequential elongations of 20:5n-3 to produce $24: 5 n-3$, which is then desaturated to $24: 6 n-3$ before being chain shortened to 22:6n-3 (Sprecher, 2000). Thus, 24:5n-3 is an important intermediate in the biosynthesis of DHA in mammals, and so the ability of fish PUFA elongases to produce 24:5n-3 through the elongation of EPA or 22:5n-3 is consistent with this pathway for DHA synthesis also operating in fish. Biochemical studies have also supported the hypothesis that DHA synthesis proceeds via $\mathrm{C}_{24}$ intermediates in fish, at least in trout (Buzzi et al., 1997).

The human elongase (ELOVL5) and rat elongase (rELO1) both also elongated monounsaturated fatty acids in addition to $\mathrm{C}_{18}$ and $\mathrm{C}_{20}$ PUFA (Leonard et al., 2000; Inagaki et al., 2002). The observation of 18:1n-7, and 20:1n-9 and 20:1n-7, in the fatty acid composition of S. cerevisiae transformed with the fish elongase ORFs in the present study indicates elongation of 16:1n-7, and also of 18:1n-9 and 18:1n-7, respectively. Therefore, these fish elongases were also able to elongate monounsaturated fatty acids in addition to PUFA, as previously demonstrated with the zebrafish and salmon elongases (Agaba et al., 2004; Hastings et al., 2004). The zebrafish elongase was also able to elongate saturated fatty acids as evidenced by the increased 18:0/16:0 ratio in transformed yeast (Agaba et al., 2004). However, this was not observed with the salmon elongase (Hastings et al., 2004), and there was no evidence that any of the fish elongases in the present study were able to elongate saturated fatty acids. Neither the mouse elov12 or human ELOVL2 clones were able to elongate saturated or monounsaturated fatty acids (Leonard et al., 2002). A plant (moss, Physcomitrella patens) PUFA elongase was cloned and characterised, and shown to be highly specific for $\Delta 6$-desaturated $\mathrm{C}_{18}$ PUFA (18:4n-3 and 18:3n-6) and possessed no activity towards saturated and monounsaturated fatty acids (Zank et al., 2002). Very recently, two types of elongase were cloned and characterised from the algae, Ostreococcus tauri and Thalassiosira pseudonana, which were specific for either $\Delta 6-\mathrm{C}_{18}$ PUFA or for $\Delta 5-\mathrm{C}_{20}$ PUFA, particularily EPA (Meyer et al., 2004).

The pattern of activity shown by the elongases from catfish and sea bream were very similar to those of the previously published zebrafish and salmon elongases (Agaba et al., 2004; Hastings et al., 2004). Tilapia was slightly unusual in that the activity towards $20: 5 n-3$ was equal to that towards 18:4n-3 and it had the highest activity towards 20:4n-6. Interestingly, the warm water species (zebrafish, catfish, tilapia and sea bream) all displayed higher activities towards the n- 6 fatty acids than 
the colder water species (salmon, turbot and cod). Turbot elongase was less active towards $\mathrm{C}_{18}$ substrates compared to almost all the other elongases. Specifically, the ability of the turbot to elongate 18:4n-3 was substantially less than that of the salmon elongase, whereas its ability to elongate 20:5n-3 was similar to that of the salmon elongase, which is consistent with earlier biochemical studies in cell lines (Ghioni et al., 1999). Only the elongase from cod was less active than that from turbot. Thus it may be that low elongase activity may be implicated in the poor ability of these marine species to synthesise n-3HUFA. The sea bream elongase on the other hand was as active as the elongases of freshwater fish, again consistent with biochemical studies in cell lines which showed that the defect in the HUFA synthesis pathway in SAF-1 (sea bream fin) cells was due to low $\Delta 5$ desaturase activity and not low elongase activity (Tocher and Ghioni, 1999). There were no obvious amino acid sequence differences between the fish species to indicate the differences in fatty acid elongation activity that were observed by heterologous expression. It was noteworthy, however, that the Kozak sequence for the turbot elongase was atypical, having a $\mathrm{C}$ three nucleotides 5' of the ATG translation initiation codon (see Table 1) that may affect translation efficiency. However, substituting the $\mathrm{C}$ with an A did not affect the pattern of activity of the turbot elongase in heterologous expression although activity may be slightly enhanced (49\% [A] v. 35\% [C] conversion of $18: 4 n-3$ to $20: 4 n-3$ and $39.3 \%$ [A] v. $30.3 \%$ [C] conversion of 20:5n-3 to 22:5n-3) (results not shown).

The study of fatty acid elongases in fish is particularly relevant at the present time with the increasing use of vegetable oils, rich in $\mathrm{C}_{18}$ PUFA, as replacements for n-3HUFA-rich fish oils in aquaculture (Sargent et al., 2002). Fatty acid compositions of fish fed vegetable oils are characterised by increased levels of $\mathrm{C}_{18}$ PUFA and decreased levels of n-3HUFA, compromising their nutritional value to the human consumer (Bell et al., 2001, 2002; Izquierdo et al., 2003; Regost et al., 2003). Therefore, there is an urgent need to optimise the ability of fish to utilise $\mathrm{C}_{18}$ PUFA and produce HUFA. Although fatty acid desaturases, such as $\Delta 6$ desaturase, are known to be crucial in HUFA synthesis, there is increasing awareness that elongases may also be points of regulation in the pathway. For instance, biochemical studies suggested that the biosynthesis of 20:4n-6 from 18:2n-6 in M. alpina may be rate-limited by the activity of the $\mathrm{C}_{18-20}$ elongase (Wynne and Ratledge, 2000). Recently, the production of AA in M. alpina was increased by metabolic engineering through the construction of a strain overexpressing the elongase (GLELO) gene (Takeno et al., 2005). Low $\mathrm{C}_{18-20}$ elongase activity had previously been observed in a turbot cell line in comparison to an Atlantic salmon cell line, suggesting it may be implicated in the inability of some marine fish to thrive on diets devoid of n3HUFA (Ghioni et al., 1999). More recently, nutritional regulation of the expression of a PUFA elongase gene was reported in Atlantic salmon, with increased expression in fish fed diets in which fish oil was replaced by linseed oil (Zheng et al., 2004b). Gene expression of the short chain fatty acid 
elongase in mice was shown to be regulated by sterol regulatory element binding protein-1 (SREBP-1) (Moon et al., 2001; Matsuzaka et al., 2002). Defects in very long chain fatty acid elongase activity have been reported in mutant (quaking and jimpy) mice (Suneja et al., 1991), and have been implicated in human disease such as Stargardt macular degeneration (Lagali et al., 2003; Karan et al., 2004). These latter examples highlight the serious consequences that deficiencies in fatty acid elongation in general can have.

In summary, full-length cDNAs for PUFA elongases were isolated from livers of several fish species. The amplified cDNA encoded ORFs of 288-294 amino acids whose sequences were highly conserved among the fish species. Heterologous expression in yeast, S. cerevisiae, demonstrated that the ORFs encoded elongases with the ability to lengthen PUFA with chain lengths from $\mathrm{C}_{18}$ to $\mathrm{C}_{22}$ and monounsaturated fatty acids, but not saturated fatty acids. Although there were no obvious amino acid sequence differences, there were differences in the functional competences of the elongases from different fish species that may contribute to the differing abilities of the species in terms of HUFA synthesis.

\section{Acknowledgements}

This work was supported partly by a Scottish Higher Education Funding Council (SHEFC) Grant (“Aquagene”) and partly by the European Union Framework V Programme (project no. QLRT-200030058, "RAFOA").

\section{References}

Agaba, M., Tocher, D. R., Dickson, C., Dick, J. R., Teale, A. J. 2004. A zebrafish cDNA encoding a multifunctional enzyme involved in the elongation of polyunsaturated, monounsaturated and saturated fatty acids. Mar. Biotechnol. 6, 251-261.

Barlow, S. 2000. Fishmeal and oil: sustainable feed ingredients for aquafeeds. Global Aquacult. Advocate 4, 85-88.

Beaudoin, F., Michaelson, L.V., Lewis, M.J., Shewry, P.R., Sayanova, O., Napier, J.A. 2000. Production of C20 polyunsaturated fatty acids (PUFAs) by pathway engineering: identification of a PUFA elongase component from Caenorhabditis elegans. Biochem. Soc. Trans. 28, 661-663.

Bell, J.G., McEvoy, J., Tocher, D.R., McGhee, F., Campbell, P.J., Sargent, J.R. 2001. Replacement of fish oil with rape seed oil in diets of Atlantic salmon (Salmo salar) affects tissue lipid compositions and hepatocyte fatty acid metabolism. J. Nutr. 131, 1535-1543. 
Bell, J.G., Henderson, R.J., Tocher, D.R., McGhee, F., Dick, J.R., Porter, A., Smullen, R., Sargent, J.R. 2002. Substituting fish oil with crude palm oil in the diet of Atlantic salmon (Salmo salar) affects tissue fatty acid compositions and hepatic fatty acid metabolism. J. Nutr. 132, 222-230.

Burr, G.O. 1981. The essential fatty acids fifty years ago. Prog. Lipid Res. 20, xxvii-xxix.

Buzzi, M., Henderson, R.J., Sargent, J.R. 1997. Biosynthesis of docosahexaenoic acid in trout hepatocytes proceeds via 24-carbon intermediates. Comp. Biochem. Physiol. 116, 263-267.

Christie, W.W. 1982. Lipid Analysis, 2nd Edn., Pergamon Press, Oxford.

Cook, H.W. 1996. Fatty acid desaturation and chain elongation in eukaryote. In: Biochemistry of Lipids, Lipoproteins and Membranes, Vance, D.E and Vance, J.E. (eds). Amsterdam: Elsevier, 129 -152 .

Folch, J., Lee, M., Sloane-Stanley, G.H. 1957. A simple method for the isolation and purification of total lipids from animal tissues. J. Biol. Chem. 226, 497-509.

Ghioni, C., Tocher, D.R., Bell, M.V., Dick, J.R., Sargent, J.R. 1999. Low $\mathrm{C}_{18}$ to $\mathrm{C}_{20}$ fatty acid elongase activity and limited conversion of stearidonic acid, 18:4n-3, to eicosapentaenoic acid, 20:5n-3, in a cell line from the turbot, Scophthalmus maximus. Biochim. Biophys. Acta 1437, 170-181.

Graham, I.A., Cirpus, P., Rein, D., Napier, J.A. 2004. The use of very long chain polyunsaturated fatty acids to ameliorate metabolic syndrome:transgenic plants as an alternative sustainable source to fish oil. Nutr. Bull. 29, 228-233.

Hastings, N, Agaba, M, Tocher, D.R., Leaver, M.J., Dick J.R., Sargent, J.R., Teale, A.J. 2001. A vertebrate fatty acid desaturase with $\Delta 5$ and $\Delta 6$ activities. Proc. Natl. Acad. Sci. U.S.A. 98, 14304 14309.

Hastings, N., Agaba, M.K., Tocher, D.R., Zheng, X., Dickson, C.A., Dick, J.R., Teale, A.J. 2004. Molecular cloning and functional characterization of fatty acyl desaturase and elongase cDNAs involved in the production of eicosapentaenoic and docosahexaenoic acids from $\alpha$-linolenic acid in Atlantic salmon (Salmo salar). Mar. Biotechnol. 6, 463-474.

Inagaki, K., Aki, T., Fukuda, Y., Kawamoto, S., Shigeta, S., Ono, K., Suzuki, O. 2002. Identification and expression of a rat fatty acid elongase involved the biosynthesis of C18 fatty acids. Biosci. Biotechnol. Biochem. 66, 613-621.

Izquierdo, M., Obach, A., Arantzamendi, L., Montero, D., Robaina, L., Rosenlund, G. 2003. Dietary lipid sources for seabream and seabass: growth performance, tissue composition and flesh quality. Aquacult. Nutr. 9, 397-407.

Karan, G., Yang, Z., Zhang, K. 2004. Expression of wild type and mutant ELOVL4 in cell culture: subcellular localization and cell viability. Molecular Vision 10, 248-253. 
Kyte, J., Doolittle, R. 1982 A simple method for displaying the hydropathic character of a protein. J. Mol. Biol. 157, 105-132.

Lagali, P.S., Liu, J., Ambasudhan, R., Kakuk, L.E., Bernstein, S.L., Seigel, G.M., Wong, P.W., Ayyagari, R. 2003. Evolutionarily conserved ELOVL4 gene expression in the vertebrate retina. Invest. Ophthalmol. Visual Sci, 44, 2841-2850.

Lands, W.E.M. 1993. Eicosanoids and health. Annals. New York Acad. Sci. 676, 46-59.

Lauritzen, L., Hansen, H.S., Jorgensen, M.H., Michaelsen, K.F. 2001. The essentiality of long chain n3 fatty acids in relation to development and function of brain and retina. Prog. Lipid Res. 40, 1-94. Leonard, A.E., Bobik, E.G., Dorado, J., Kroeger, P.E., Chuang, L.-T., Thurmond, J.M., Parker-Barnes, J.M., Das, T., Huang Y.-S., Murkerji, P. 2000. Cloning of a human cDNA encoding a novel enzyme involved in the elongation of long chain polyunsaturated fatty acids. Biochem. J. 350, 765770 .

Leonard, A.E., Kelder, B., Bobik, E.G., Chuang, L.-T., Lewis, C.J., Kopchick, J.J., Murkerji, P., Huang, Y.-S. 2002. Identification and expression of mammalian long-chain PUFA elongation enzymes. Lipids 37, 733-740.

March, B.E. 1993. Essential fatty acids in fish physiology. Can. J. Physiol. Pharmacol. 71, 684-689.

Matsuzaka, T., Shimano, H., Yahagi, N., Yoshikawa, T., Amemiya-Kudo, M., Hasty, A.H., Okazaki, H., Tamura, Y., Iizuka, Y., Ohashi, K., Osuga, J., Takahashi, A., Yato, S., Sone, H., Ishibashi, S., Yamada, N. 2002. Cloning and characterization of a mammalian fatty acyl-CoA elongase as a lipogenic enzyme regulated by SREBPs. J. Lipid Res. 43, 911-920.

Meyer, A., Kirsch, H., Domergue, F., Abbadi, A., Sperling, P., Bauer, J., Cirpus, P., Zank, T.K., Moreau, H., Roscoe, T.J., Zähringer, U., Heinz, E. 2004. Novel fatty acid elongases and their use for the reconstitution of docosahexaenoic acid biosynthesis. J. Lipid Res. 45, 1899-1909.

Moon, Y., Shah, N.A., Mohapatra, S., Warrington, J.A., Horton, J.D. 2001. Identification of a mammalian long chain fatty acyl elongase regulated by sterol regulatory element-binding proteins. J. Biol. Chem. 276, 45358-45366.

Mourente, G., Tocher, D.R. 1993a. Incorporation and metabolism of ${ }^{14} \mathrm{C}$-labelled polyunsaturated fatty acids by juvenile gilthead sea bream Sparus aurata L. in vivo. Fish Physiol. Biochem. 10, 443453.

Mourente, G., Tocher, D.R. 1993b. Incorporation and metabolism of ${ }^{14}$ C-labelled polyunsaturated fatty acids by wild-caught juveniles of golden grey mullet Liza aurata, in vivo. Fish Physiol. Biochem. 12, 119-130. 
Mourente, G., Tocher, D.R. 1994. In vivo metabolism of $\left[1-{ }^{14} \mathrm{C}\right]$ linolenic acid (18:3n-3) and [1${ }^{14}$ C] eicosapentaenoic acid $(20: 5 n-3)$ in a marine fish. Time course of the desaturation/elongation pathway. Biochim. Biophys. Acta 1212, 109-118.

Nelson, J.S. 1994. Fishes of the World. 3rd Ed. John Wiley and Sons, New York, N.Y.

Nugent, A.P. 2004. The metabolic syndrome. Nutr. Bull. 29, 36-43.

Owen, J.M., Adron, J.A., Middleton, C., Cowey, C.B. 1975. Elongation and desaturation of dietary fatty acids in turbot (Scophthalmus maximus) and rainbow trout (Salmo gairdneri). Lipids 10, 528531.

Parker-Barnes, J.M., Das,T., Bobik, E., Leonard, A.E., Thurmond, J.M., Chuang, L., Huang, Y.S., Mukerji, P. 2000. Identification and characterization of an enzyme involved in the elongation of n6 and n-3 polyunsaturated fatty acid. Proc. Natl. Acad. Sci. USA 97, 8284-8289.

Qui, X., Hong, H., MacKenzie, S.L. 2001. Identification of a $\Delta 4$ fatty acid desaturase from Thraustochytrium $s p$ involved in the synthesis of docosahexaenoic acid by heterologous expression in Saccharomyces cerevisiae and Brassica juncea. J. Biol. Chem. 276, 31561-31566.

Regost, C., Arzel, J., Robin, J., Rosenlund, G., Kaushik, S.J. 2003. Total replacement of fish oil by soybean or linseed oil with a return to fish oil in turbot (Psetta maxima). 1. Growth performance, flesh fatty acid profile, and lipid metabolism. Aquaculture 217, 465-482.

Saitou, N., Nei, M. 1987. The neighbor-joining method. A new method for reconstructing phylogenetic trees. Mol. Biol. Evol. 4, 406-425.

Sargent, J.R., Bell, J.G., Bell, M.V., Henderson, R.J., Tocher , D.R. (1995) Requirement criteria for essential fatty acids. J. Appl. Ichthyol. 11, 183-198.

Sargent, J.R., Tocher, D.R., Bell, J.G. 2002. The lipids. In: Fish Nutrition. Third edition, Halver, J. E. and Hardy, R.W. (eds). San Diego:Academic Press, 181-257.

Simopoulos, A.P. 2000. Human requirements for n-3 polyunsaturated fatty acids. Poult. Sci. 79, 961970.

Sprecher, H. 2000. Metabolism of highly unsaturated n-3 and n-6 fatty acids. Biochim Biophys. Acta $1486,219-231$.

Suneja, S.K., Nagi, M., Cook, L., Cinti, D. 1991. Decreased long-chain fatty acyl-CoA elongation activity in quaking and jimpy mouse brain: Deficiency in one enzyme or multiple enzyme activities. J. Neurochem. 57, 140-146.

Takeno, S., Sakuradani, E., Murata, S., Inohara-Ochiai, M., Kawashima, H., Ashikari, T., Shimizu, S. 2005. Molecular evidence that the rate-limiting step for the biosynthesis of arachidonic acid in Mortierella alpina is at the level of an elongase. Lipids 40, 25-30. 
Tidwell, J.H., Allan, G.L. 2002. Fish as food: aquaculture's contribution. World Aquaculture 33, 4448.

Tocher, D.R. 2003. Metabolism and functions of lipids and fatty acids in teleost fish. Rev. Fisheries Sci. 11, 107-184.

Tocher, D.R., Ghioni, C. 1999. Fatty acid metabolism in marine fish: Low activity of $\Delta 5$ desaturation in gilthead sea bream (Sparus aurata) cells. Lipids 34, 433-440.

Tocher, D. R., Agaba, M., Hastings, N., Bell, J. G., Dick, J. R., Teale, A. J. 2001. Nutritional regulation of hepatocyte fatty acid desaturation and polyunsaturated fatty acid composition in zebrafish (Danio rerio) and tilapia (Oreochromis nilotica). Fish Physiol. Biochem. 24, 309-320.

Tocher, D.R., Leaver, M.J., Hodgson, P.A. 1998. Recent advances in the biochemistry and molecular biology of fatty acyl desaturases. Prog. Lipid Res. 37, 73-117.

Tvrdik, P., Westerberg, R., Silve, S., Asadi, A., Jakobsson, A., Cannon, B., Loison, G., Jacobsson, A. 2000. Role of a new mammalian gene family in the biosynthesis of very long chain fatty acids and sphingolipids. J. Cell Biol. 149, 707-717.

Wynn, J.P.. Ratledge, C. 2000. Evidence that the rate limiting step for the biosynthesis of arachidonic acid in Mortierella alpina is at the level of the 18:3 to 20:3 elongase. Microbiology 146, 23252331.

Zank, T.K., Zaehringer, U., Beckmann, C., Pohnert, G., Boland, W., Holtorf, H., Reski, R., Lerchl, J., Heinz, E. 2002. Cloning and functional characterisation of an enzyme involved in the elongation of delta 6-polyunsaturated fatty acids from the moss Physcomitrella patens. Plant J. 31, 255-268.

Zheng, X., Seiliez, I., Hastings, N., Tocher, D.R., Panserat, S., Dickson, C.A., Bergot, P., Teale, A.J. 2004a. Characterization and comparison of fatty acyl $\Delta 6$ desaturase cDNAs from freshwater and marine teleost fish species. Comp. Biochem. Physiol. 139B, 269-279.

Zheng, X., Tocher, D.R., Dickson, C.A., Bell, J.G., Teale, A.J. 2004b. Effects of diets containing vegetable oil on expression of genes involved in polyunsaturated fatty acid biosynthesis in liver of Atlantic salmon (Salmo salar). Aquaculture 236, 467-483. 
Legends to Figures.

Fig.1. Pathways of highly unsaturated fatty acid (HUFA) biosynthesis from the $\mathrm{C}_{18}$ polyunsaturated fatty acids (PUFA), 18:3n-3 and 18:2n-6. Solid lines represent steps that have been shown to occur in fish. Dotted lines represent steps that have not been directly demonstrated in fish. $\Delta 6, \Delta 5$ and $\Delta 4$, fatty acid desaturases; Elo, fatty acid elongases; Short, peroxisomal chain shortening.

Fig.2. Comparison of the deduced amino acid sequence of fatty acyl elongases from catfish, tilapia, turbot, seabream and cod with that of elongases from zebrafish, salmon and human ELOVL5. Identical residues are shaded black and similar residues are shaded grey. Identity/similarity shading was based on the BLOSUM62 matrix and the cut off for shading was 75\%. Motifs highly conserved among fatty acid elongases from a wide range of organisms are boxed. Transmembrane regions (not indicated) were predicted from a hydropathy plot taking peaks with scores $>1.6$ using a scan window size of 18 (Kyte and Doolittle, 1982).

Fig.3. Phylogenetic tree comparing amino acid sequences of elongases from catfish, tilapia, turbot, seabream and cod with those of elongases from other fish species (zebrafish and salmon), mammals (human, mouse and rat), bird (chicken, Gallus gallus) amphibian (Xenopus laevis), insect (Drosophila melanogaster), fungus (M. alpina), nematode (C.elegans) and plant (moss, Physcomitrella patens). The tree was constructed using the N-J method using CLUSTALX and NJPLOT (Saitou and Nei, 1987). The horizontal branch length is proportional to the amino acid substitution rate per site. The numbers represent the frequencies with which the tree topology presented here was replicated after 1000 bootstrap iterations.

Fig.4. Identification of fatty acid elongation products in transgenic yeast (S. cerevisiae) grown in the presence of 18:4n-3. Fatty acids were extracted from yeast transformed with pYX222 vector without insert (A) or containing the putative fatty acid elongase cDNA inserts from catfish (B), tilapia (C), seabream (D), turbot (E) and cod (F). The first four peaks in each panel are the main endogenous fatty acids of S. cerevisiae, namely 16:0 (1), 16:1n-7 (2), 18:0 (3) and 18:1n-9 (4), and peak 6 is the exogenously added substrate fatty acid, 18:4n-3. The main additional peaks in panels B-F represent elongated products of 18:4n-3, specifically 20:4n-3 (9) and 22:4n-3 (10). Further additional peaks observed were 18:1n-7 (5), 20:1n-9 (7) and 20:1n-7 (8). Vertical axis, FID response; horizontal axis, retention time. 
Fig.5. Identification of fatty acid elongation products in transgenic yeast (S. cerevisiae) grown in the presence of 20:5n-3. Fatty acids were extracted from yeast transformed with pYX222 vector without insert (A) or containing the putative fatty acid elongase cDNA inserts from catfish (B), tilapia (C), seabream (D), turbot (E) and cod (F). Peaks 1-5, 7 and 8 are as described in the legend to Fig.4. Peak 11 is the exogenously added substrate fatty acid, 20:5n-3, and the main additional peak in panels B-F represents 22:5n-3 (12) the elongated product of 20:5n-3. Vertical axis, FID response; horizontal axis, retention time.

Fig.6. Identification of fatty acid elongation products in transgenic yeast (S. cerevisiae) grown in the presence of 22:5n-3. Fatty acids were extracted from yeast transformed with pYX222 vector without insert (A) or containing the putative fatty acid elongase cDNA inserts from catfish (B) and seabream (C). Peaks 1-5, 7, 8 and 12 are as described in the legend to Fig.5. and the additional peak in panels B and $C$ represent 24:5n-3 (13) the elongated product of 22:5n-3. Vertical axis, FID response; horizontal axis, retention time.

Fig.7. Identification of fatty acid elongation products in transgenic yeast (S. cerevisiae) grown in the presence of n-6 fatty acids. Fatty acids were extracted from yeast transformed with pYX222 vector without insert (A and $\mathrm{C}$ ) or containing the putative fatty acid elongase cDNA insert from cod (B and D). Peaks 1-5 and 7 are as described in the legend to Fig.4. Peak 14 in panels A and B represents 18:3n-6, while peak 16 in panels $C$ and D represents 20:4n-6 (14), the exogenously added substrate fatty acids. The additional peak in panels B and D are 20:3n-6 (15) and 22:4n-6 (17), the respective elongated products of 18:3n-6 and 20:4n-6. Vertical axis, FID response; horizontal axis, retention time. 
Table 1. Forward (sense) and reverse (antisense) primers used for heterologous expression

\begin{tabular}{|c|c|c|c|}
\hline Species & Direction & Code & Primer sequence \\
\hline \multirow[t]{2}{*}{ Catfish } & Sense & CatEloxA & $5^{\prime}$ aagaattcaaaatggaaatctttaaccacag $3^{\prime}$ \\
\hline & Antisense & CatEloxB & 5' cataactcgagaatgtacaggtcctcagtcgec 3 , \\
\hline \multirow[t]{2}{*}{ Tilapia } & Sense & OniEloxA & $5^{\prime}$ aagaattcaagctacggtgaaaaatggagacc $3^{\prime}$ \\
\hline & Antisense & OniEloxB & 5' cataactcgagtctcaaatgtcaatccaccc 3' \\
\hline \multirow[t]{2}{*}{ Turbot } & Sense & TurbEloxA & 5' aagaattcaagcttggaactttctggtgacaaatg 3' \\
\hline & Antisense & TurbEloxB & 5' aactcgagcaaatgtcaatccaccctcagc 3' \\
\hline \multirow[t]{2}{*}{ Sea bream } & Sense & SauEloxA & $5^{\prime}$ aaagaattcaaagggtgaaagatggagacc $3^{\prime}$ \\
\hline & Antisense & SauEloxB & $5^{\prime}$ ataactcgagacagtgagaatggggtgacg $3^{\prime}$ \\
\hline \multirow[t]{2}{*}{ Cod } & Sense & CodeloxA & 5 ' taagaattcggtgaaaaatggagccattcaatca 3 , \\
\hline & Antisense & CodEloxB & $5^{\prime}$ ataactcgagtgtacatcaatccaccetcagc $3^{\prime}$ \\
\hline
\end{tabular}

The forward primers contain the Kozak translation initiation sequence (boldface' and an EcoRI site (underlined). The reverse primers flank or contain the termination codon and have a XhoI site (underlined). 
Table 2. Identity matrix showing the results of a pair-wise comparison between the identities of th amino acid sequences of the fish and human fatty acid elongases.

\begin{tabular}{lccccccc}
\hline & Catfish & Tilapia & Salmon & Turbot & Seabream & Cod & Human \\
\hline Zebrafish & 76.6 & 69.6 & 75.0 & 72.3 & 74.4 & 73.9 & 67.8 \\
Catfish & - & 68.0 & 74.6 & 73.0 & 73.0 & 79.4 & 67.5 \\
Tilapia & - & - & 75.0 & 77.2 & 79.3 & 71.1 & 61.2 \\
Salmon & - & - & - & 80.7 & 81.4 & 75.0 & 67.8 \\
Turbot & - & - & - & - & 86.0 & 75.5 & 65.2 \\
Seabream & - & - & - & - & - & 77.2 & 68.2 \\
Cod & - & - & - & - & - & - & 65.5 \\
\hline
\end{tabular}

Data are percentages of amino acid residues that are identical. 
Table 3. Functional characterisation of fish fatty acyl elongase cDNAs

\begin{tabular}{ccccccccccccccc}
\hline & \multirow{2}{*}{ Zebrafish } & \multicolumn{2}{c}{ Catfish } & \multicolumn{2}{c}{ Tilapia } & \multicolumn{2}{c}{ Salmon } & \multicolumn{2}{c}{ Turbot } & \multicolumn{2}{c}{ Sea bream } & \multicolumn{2}{c}{ Cod } \\
& $\mathrm{n}-3$ & $\mathrm{n}-6$ & $\mathrm{n}-3$ & $\mathrm{n}-6$ & $\mathrm{n}-3$ & $\mathrm{n}-6$ & $\mathrm{n}-3$ & $\mathrm{n}-6$ & $\mathrm{n}-3$ & $\mathrm{n}-6$ & $\mathrm{n}-3$ & $\mathrm{n}-6$ & $\mathrm{n}-3$ & $\mathrm{n}-6$ \\
\hline $\mathrm{C}_{18-20}$ & 85.4 & 70.7 & 87.5 & 78.3 & 82.6 & 71.2 & 71.8 & 42.1 & 34.6 & 36.6 & 85.9 & 84.3 & 7.4 & 15.7 \\
$\mathrm{C}_{20-22}$ & 49.4 & 25.6 & 48.1 & 40.8 & 86.6 & 61.8 & 38.9 & 22.6 & 30.3 & 18.1 & 66.8 & 43.9 & 0.8 & 1.5 \\
$\mathrm{C}_{22-24}$ & 4.9 & 0.5 & 2.8 & 0.9 & 1.0 & $\operatorname{tr}$ & 0.7 & 0.3 & 0.6 & 0.6 & 4.7 & 0.8 & $\mathrm{nd}$ & $\mathrm{nd}$ \\
\hline
\end{tabular}

Results are presented as percentage of substrate fatty acid converted to elongated product.

Fatty acid substrates were; $\mathrm{C}_{18-20}, 18: 4 n-3$ and 18:3n-6; $\mathrm{C}_{20-22}, 20: 5 n-3$ and 20:4n-6; $\mathrm{C}_{22-24}, 22: 5 n-3$ and 22:4n-6. nd, not detected; $\operatorname{tr}$, trace $(<0.05 \%)$. 
18:3n-3

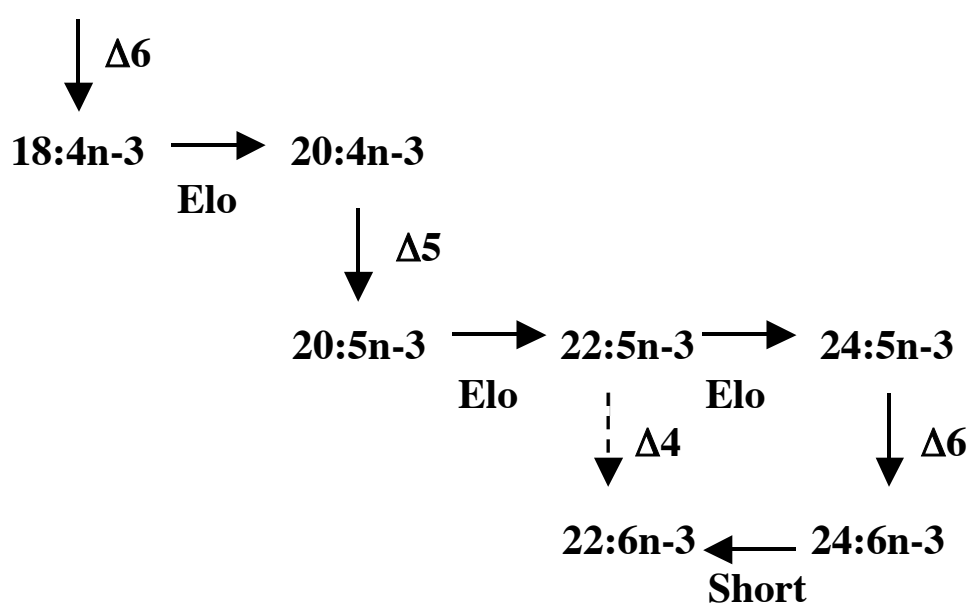

Elo

18:2n-6

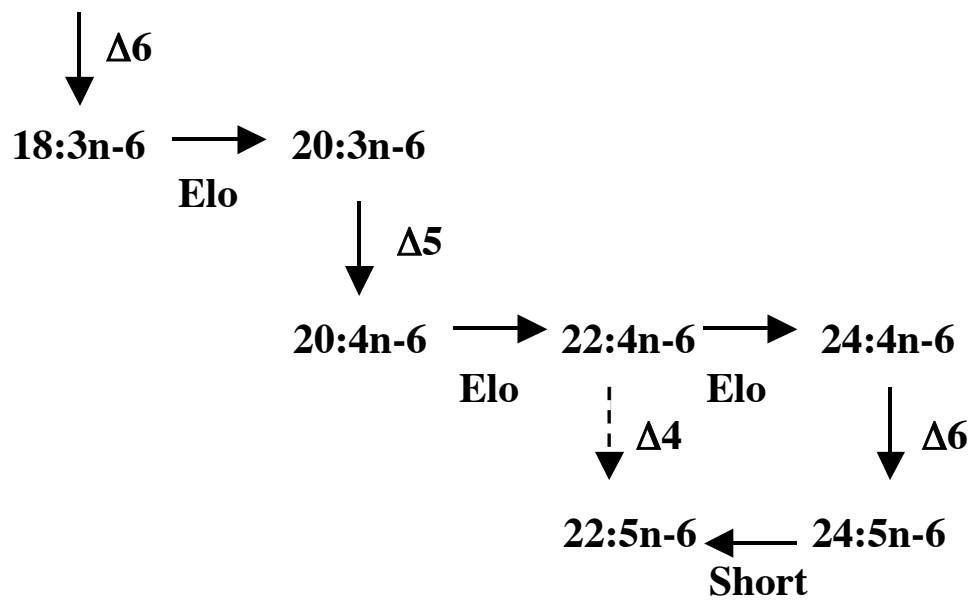


Fig. 2 
Zebrafish

Catfish

Tilapia

Salmon

Turbot

Sea bream

cod

HumanELOVL5

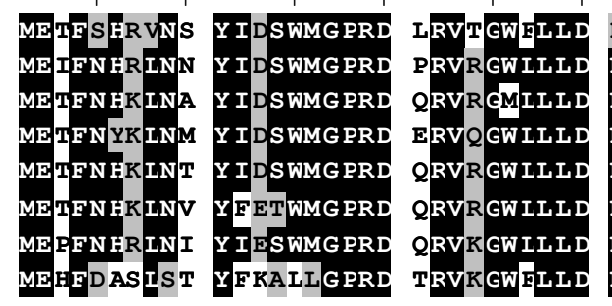

50
50
50
50
50
50
50
50

Zebrafish
Catfish
Tilapia
Salmon
Turbot
Sea bream
Cod
HumanELOVL5

Zebrafish

Catfish

Tilapia

Salmon

Turbot

Sea bream

Cod

HumanELOVL5
60
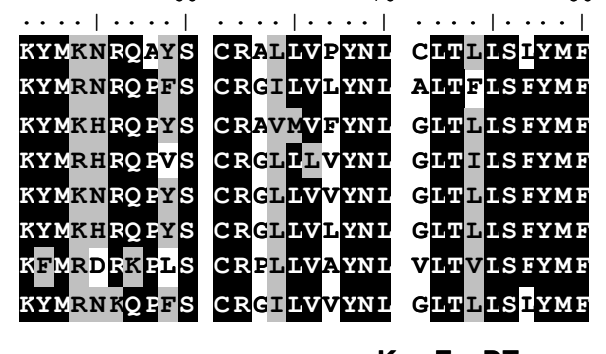

80

90

100
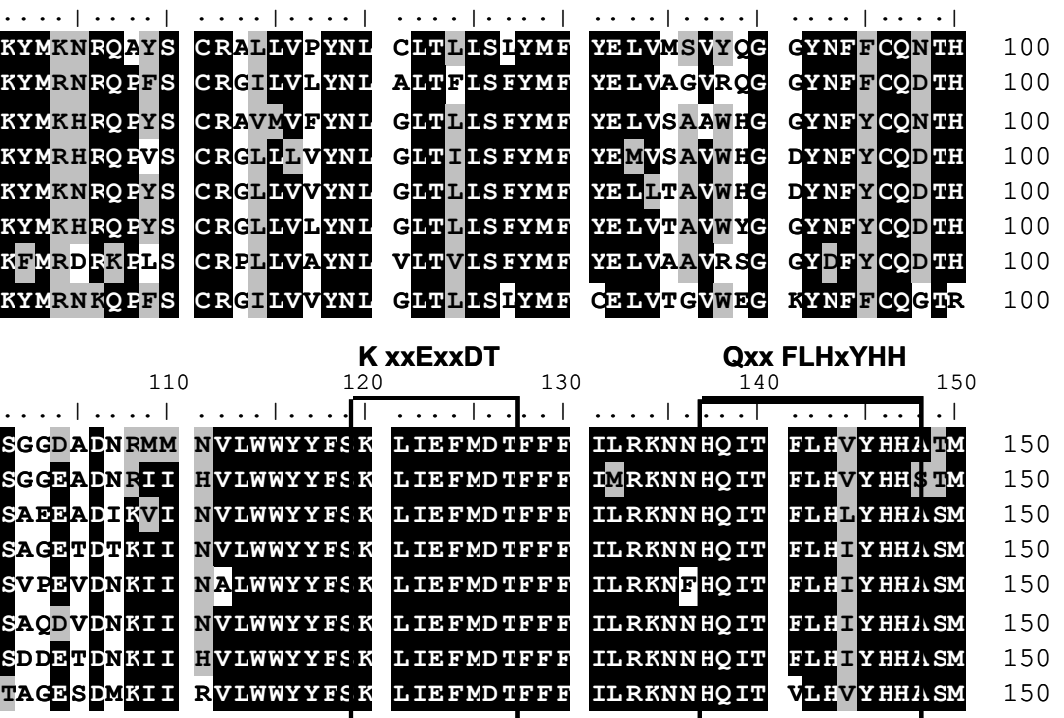

TESDII RVLWWYYFS K LIEFMDTE

100

100

100

100

100

100
Zebrafish
Catfish
Tilapia
Salmon
Turbot
Sea bream
Cod
HumanELOVL5

Zebrafish

Catfish

Tilapia

Salmon

Turbot

Sea bream

Cod

HumanELOVL5

Zebrafish

Catfish

Tilapia

Salmon

Turbot

Sea bream

Cod

HumanELOVL5
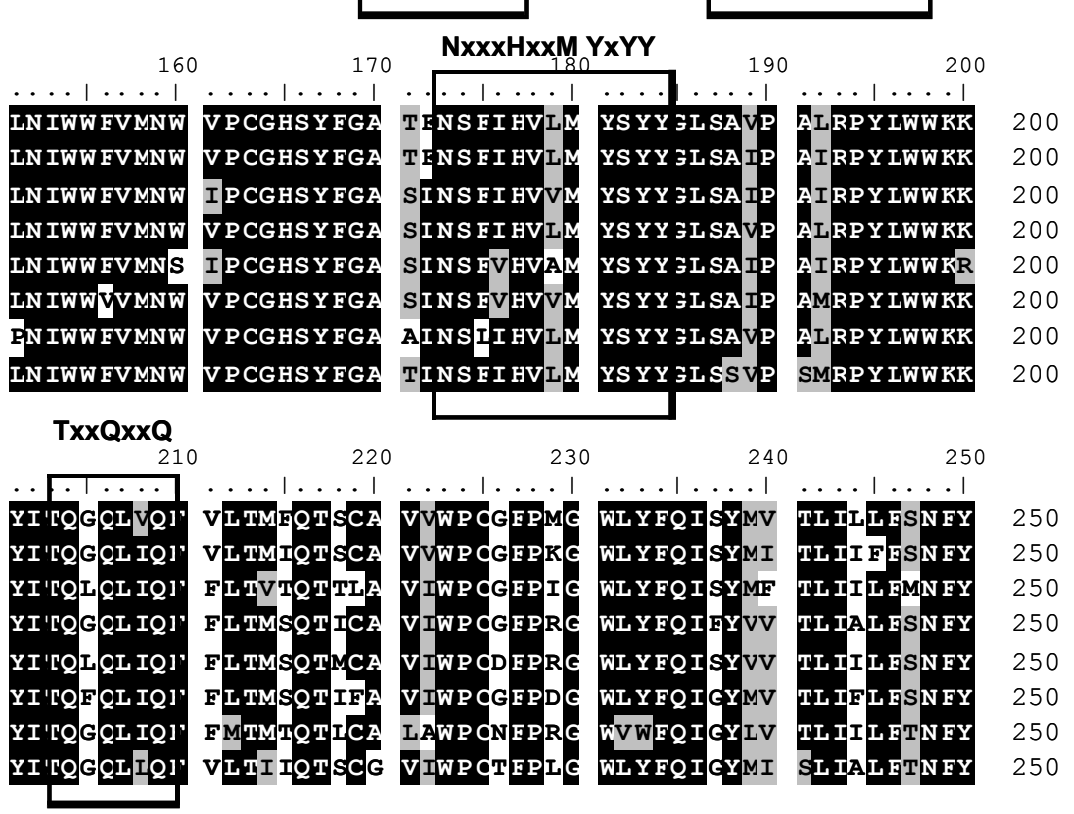

260

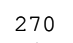

280

290

IQTYKKRSGS RKS----DYP N---GSVNGF INGVMSSEK- IKHRKARAD 291 KQTYKKRGDA LKS----DHR HNAVKSVNGH SNGTTHKET- VKQRKRRGD 294 IQTY-KKSGS QRK----HPQ NSSLISTNGF ANGTPSTDF- TAPKKIRVD 293 IQTYKKHLVS QKKE---CHQ NGSVASINGF VNGVTPTVT- ITHRKVRGD 295 IQTYKKHNAT LQK----QHP NGSAVSRNGH SNGTPSAEH- MAHKKIRVD 294 MQTYNKHSAS LRK----EHQ NGSPISTNGF ANGTPSMEH- AAHKKIRVD 294 IQTYKKQKVS LKN------- -GS--STNGF ANGVSHVEH- SLHKKLRVD 288 IQTYNKKGAS RRKDHLKDHQ NGSMAAVNGF TNSFSPLENN VKPRKIRKD 299 
Fig.3.

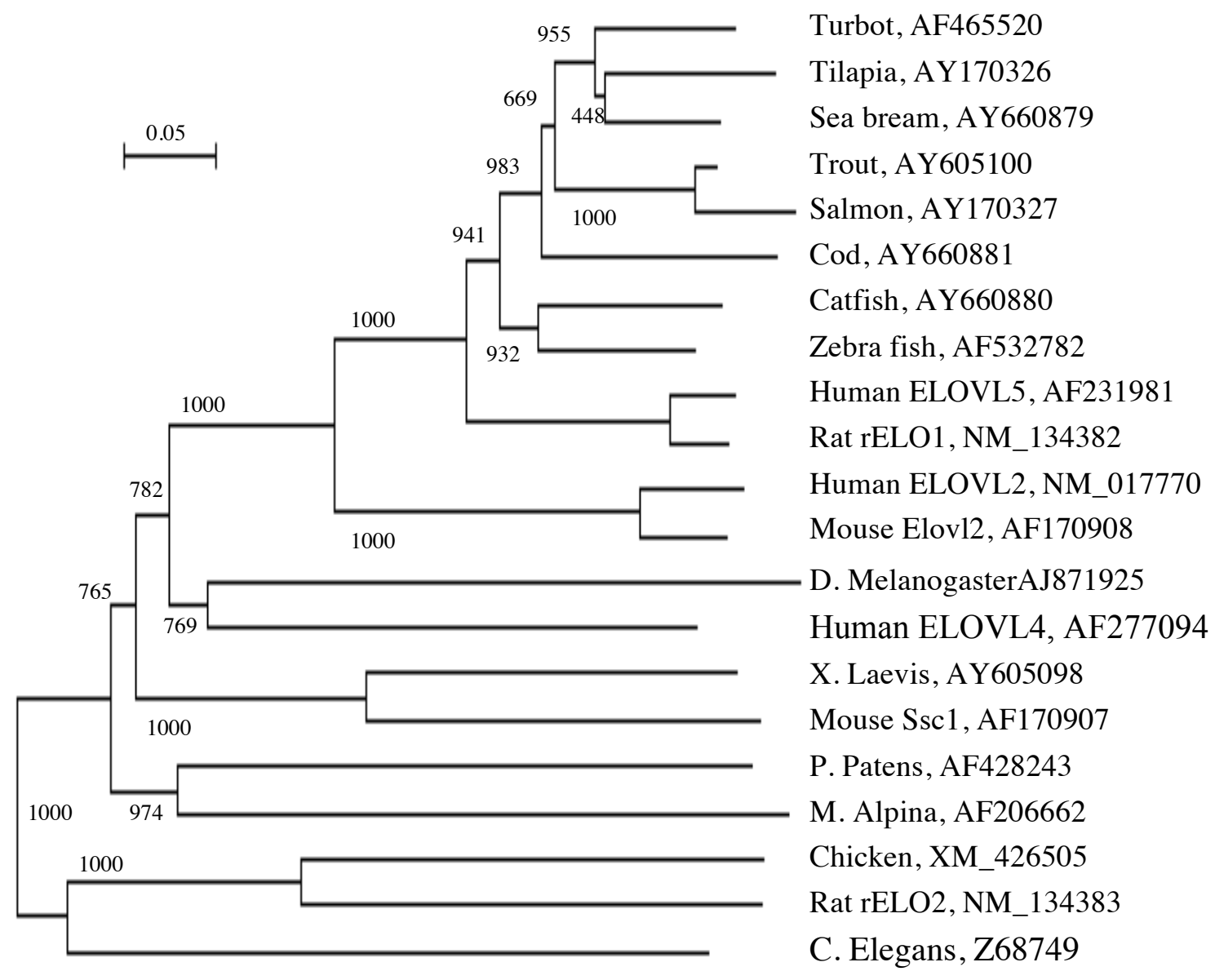




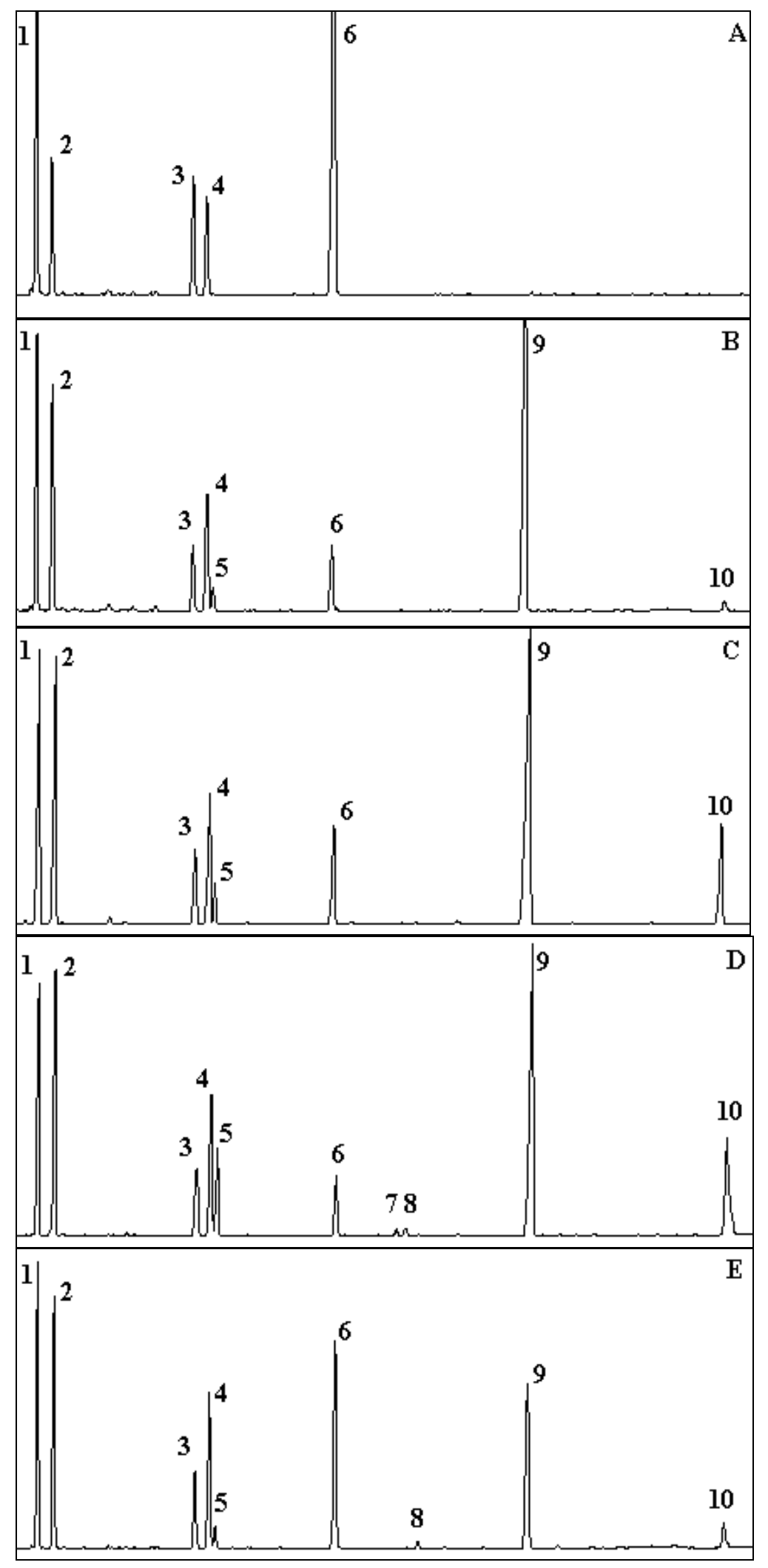




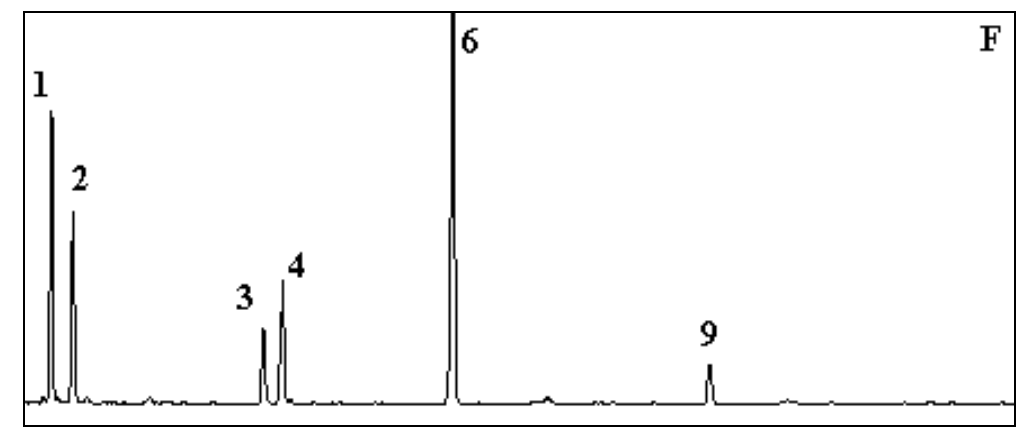



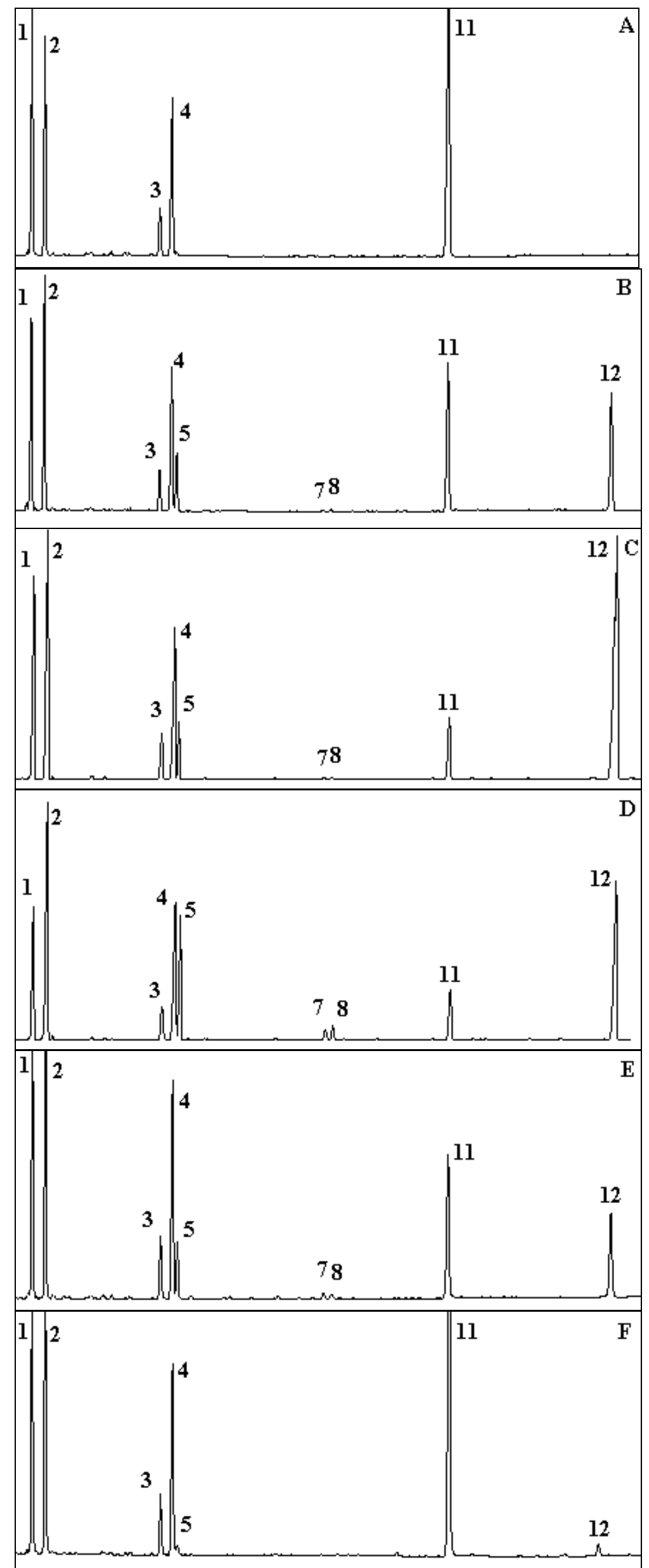


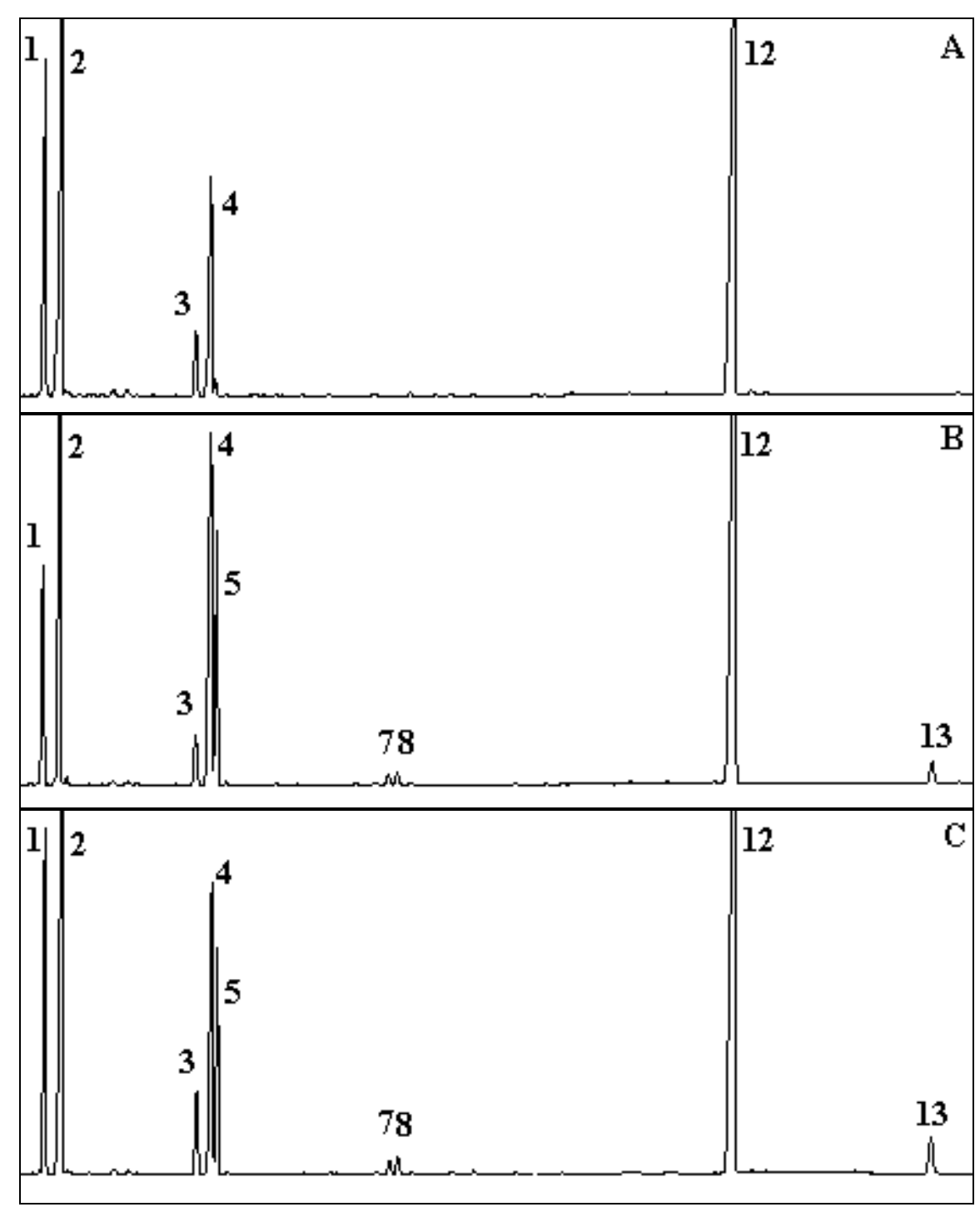



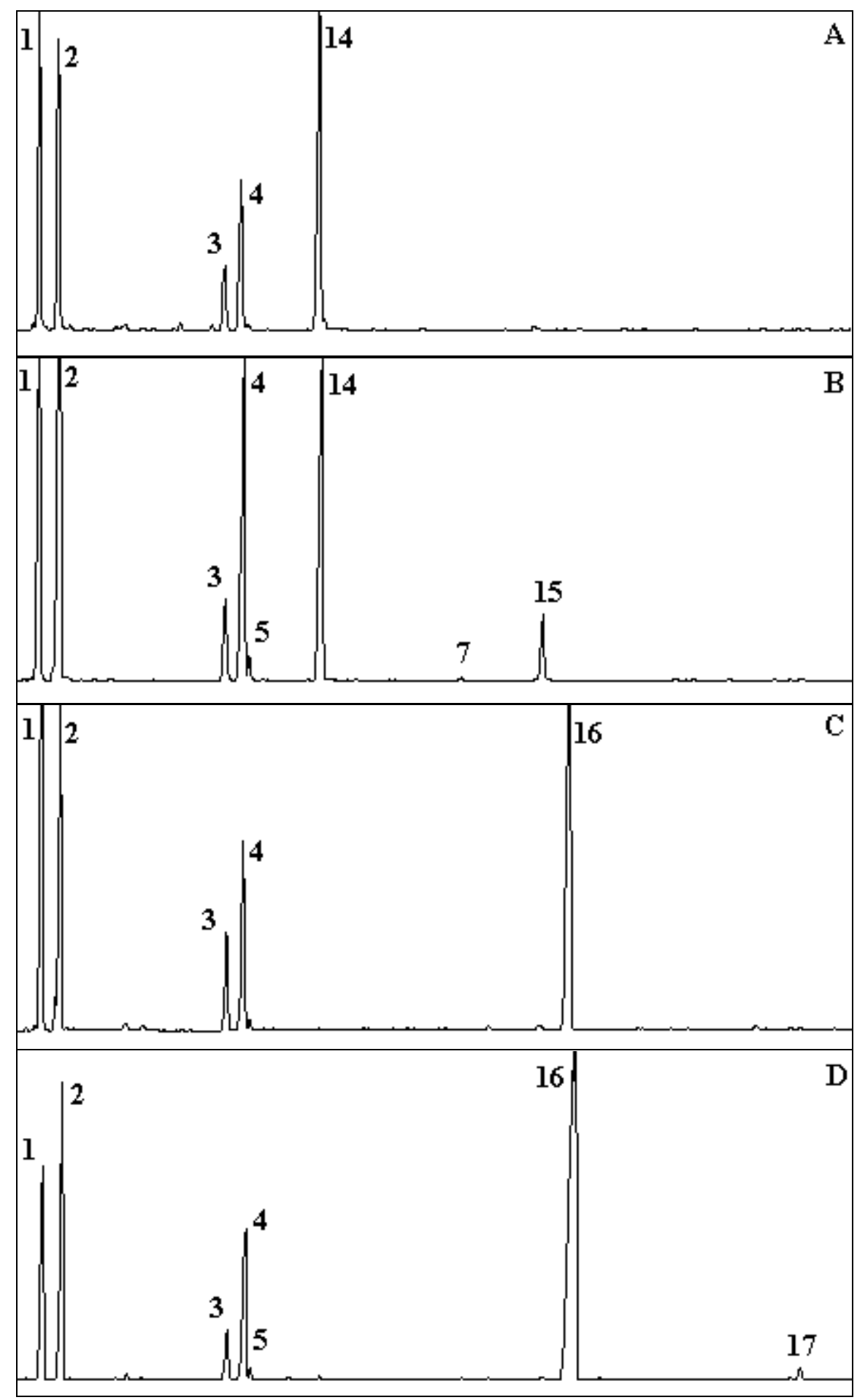